\title{
La formación humanista en educación superior. Programas de tutorías en las universidades
}

\section{Humanistic learning in higher education. Tutoring programs at universities A formação humanista em educação superior. Programas de tutoria nas universidades}

\author{
Leticia Sesento García \\ Colegio Primitivo y Nacional de San Nicolás de Hidalgo (UMSNH) \\ Michoacán, México \\ leticiasesentogarcia@yahoo.com.mx \\ (D) https://orcid.org/0000-0002-6456-058X
}

Recibido - Received - Recebido: 07 / 10 / 2020 Corregido - Revised - Revisado: 23 / 04 / 2021 Aceptado - Accepted - Aprovado: 25 / 05 / 2021

\begin{abstract}
Resumen: En el presente ensayo se reconoce a la educación humanista como una estrategia pertinente para la educación superior universitaria. La educación humanista es uno de los aspectos fundamentales para el proceso de formación en el nivel superior para lograr una sociedad más justa. Por ello, al trabajar con el estudiantado se requiere aplicar métodos educativos que enseñen a una persona a tener un determinado código de conducta con él mismo y los demás. Papalia, Martorell y Feldman (2006) señalan la necesidad de reconocer el respeto a las reglas para concertar a una sociedad justa y participativa. Para el devenir de la educación, es necesario recuperar el enfoque de la formación humanista en la universidad. El humanismo se concibe como un proceso selecto para el establecimiento de relaciones más humanas, transformaciones dialógicas y métodos de colaboración, por medio del ejercicio colectivo entre semejantes; desde luego, con la invaluable ayuda de los profesores. Es necesario que esta idea humanista de la educación se refleje en la cotidianidad del aula escolar; no se trata que sea una teoría que analicen las academias de profesores universitarios, sino este enfoque sea llevado de manera práctica en cada relación o interacción del profesorado con su estudiantado. Además, se analiza la educación en valores como elemento fundamental en esta época; la educación integral es una peculiaridad pedagógica en todos los procesos de formación humana.
\end{abstract}

Palabras claves: Educación humanista, Universidad, Docente, Tutoría, Estudiante, Educación, Enseñanza superior

Summary: In this essay, humanistic education is recognized as a pertinent strategy for university higher education. Humanistic education is one of the fundamental aspects for the training process at the higher level to achieve a fairer society. Therefore, when working with the student body, it is necessary to apply educational methods that teach a person to have a certain code of conduct with himself and others. Papalia, Martorell and Feldman (2006) point out the need to recognize respect for the rules in order to establish a just and participatory society. For the future of education, it is necessary to recover the focus of humanistic training at the university. Humanism is conceived as a select process for the establishment of relationships that are more human, dialogic transformations and collaboration methods, through the collective exercise between peers and certainly with the invaluable help of the teachers. It is necessary that this humanistic idea of education be reflected in the daily life of the school classroom. It is not intended to be a theory analyzed by the academia of university professors, but rather this approach is carried out in a practical way in each relationship or interaction of the teaching staff with their students. In addition, education in values is analyzed as a fundamental element at this time. Comprehensive education is a pedagogical peculiarity in all processes of human education.

Keywords: Humanistic education, University, Teacher, Tutoring, Student, Education, Higher education 
Resumo: No presente ensaio se reconhece a educação humanista como uma estratégia pertinente para a educação superior universitária. A educação humanista é um dos aspectos fundamentais para o processo de formação em um nível superior para atingir uma sociedade mais justa. Por isso, ao trabalhar com um corpo estudantil é necessário aplicar métodos educacionais que ensinem a uma a pessoa a ter um determinado código de conduta com si mesma e com os demais. Papalia, Martorell y Feldman (2006) salientam a necessidade de reconhecer o respeito às regras para assentar uma sociedade justa e participativa. Para o futuro da educação, é necessário recuperar o foco da formação humanística na universidade. O humanismo é concebido como um processo seletivo para o estabelecimento de relações mais humanas, transformações dialógicas e métodos de colaboração, por meio do exercício coletivo entre pares; certamente com a ajuda inestimável dos professores. É necessário que esta ideia humanista da educação seja refletida na cotidianeidade das salas aula; não para que se transforme em uma teoria que seja analisada pela esféra acadêmica de professores universitários, se não que esta abodagem seja tida de maneira prática em cada relação ou interação do professorado com o corpo de estudantes. Além disso, é analisada a educação enquadrada em valores como elemento fundamental nesta época; a educação intengral é uma peculiaridade pedagógica em todos os processo de formação humana.

Palavras chave: Educação humanista, universidade, docente, tutoria, estudante, educação, ensino superior

\section{INTRODUCCIÓN}

El propósito de este manuscrito es destacar algunas premisas esenciales del paradigma pedagógico denominado como humanismo. En el campo educativo, el interés de los humanistas se centra en proponer una educación integral para alcanzar un desarrollo total de la persona, para alcanzar la autorrealización del alumnado. La persona estudiante es un ser único, irrepetible con necesidades personales de crecimiento y tendrá que ser visto como persona no como sujeto fragmentado.

Desde esta perspectiva pedagógica, la persona docente se percibe como un guía y un facilitador de las potencialidades que poseen los educandos. Las actividades que realicen deben estar encaminados a fortalecer el autoaprendizaje y la creatividad. Hoy existen innumerables estudios, de diferentes índoles, sobre el paradigma humanista y su relación con la educación. Sin embargo, lo que se propone en el presente trabajo es una revisión del paradigma pedagógico humanista de cómo trabajarlo en el aula con el estudiantado.

La educación humanista, así como las estrategias de acompañamiento en el nuevo paradigma educativo y su impacto en los procesos centrados en los aprendizajes de los estudiantes, se ha convertido en la última década, en objeto de análisis y estudio. Los constantes cambios y transformaciones de la educación ante el nuevo paradigma formativo, dirigido al aprendizaje de los estudiantes, exige reflexionar sobre los métodos de orientación, el desarrollo de conocimientos actitudinales y valóricos, que son aspectos medulares en la educación integral de las personas estudiantes.

Las instituciones de educación superior, esencialmente las universidades públicas, tienen el reto de ubicar sus métodos pedagógicos hacia conocimientos y aprendizajes enfocados a la formación de recursos humanos calificados tanto como profesionistas, así como personas como mecanismo clave para incidir en el desarrollo al país. En este reto, las Instituciones de Educación Superior (IES), tendrán los siguientes desafíos: reducir el fracaso escolar, la deserción escolar y el abandono definitivo de la universidad, por parte de algunos estudiantes, cuya salida y abandono suele marcarle para el resto de su existencia. De ahí el acento de este artículo en la construcción de programas de atención que admitan fortalezcan la formación humanista, pero destacando siempre el papel fundamental del profesor universitario.

Por tal motivo, es necesario reflexionar en torno a las funciones del profesorado en la formación humanista como estrategia para la formación integral de calidad ante el nuevo paradigma educativo centrado en el estudiantado y plantear elementos claves para el perfeccionamiento de estrategias oportunas y exitosas.

Las transformaciones que se han desarrollado actualmente, toman rasgos de una nueva sociedad con base en el conocimiento; sin embargo, resulta necesario cimentarlas en premisas humanistas y la 
formación en valores, con sujetos críticos egresados con orgullo de una universidad. Es importante señalar que un conocimiento relacionado sustancialmente con la ética será el medio para facilitar un mundo más igualitario. La nueva sociedad del conocimiento corresponderá relacionarse con los aspectos socio-afectivos que favorecen el éxito escolar.

Con frecuencia, la formación humanista se enseña de manera similar como se enseñan los contenidos disciplinares y el resultado inmediato es una "intelectualización", todo dispositivo cognitivo (conocimiento) suele tener como resultado que no llegue a la parte sensible del ser humano. Es preciso pensar en términos científicos e intelectuales también, pero relacionarlo con el mecanismo afectivo (emociones). Como lo establecía el educador Alexander S. Neill, (1979), cuando sostenía que "corazones, no solo cabezas en la escuela". Los valores humanistas agudizan los sentidos en cuanto a las acciones de los sujetos en el devenir cotidiano de su vida, cada individuo debe instituir su apropiado esquema humanista. La función de los profesores, en este contexto, es beneficiar el proceso, permitir y desdoblar atmósferas en el entorno de los estudiantes para que vivan y aprecien los valores fundamentales, y de esta manera, sean interiorizados por ellos.

La educación humanista se ocupa de los espacios morales de las personas, para proporcionar terreno a la independencia, al uso del diálogo en todo proceso de comunicación. Al mismo tiempo, se constituyen en un habilitador en la cimentación de elementos y políticas que, al sostener un humanismo como base, podrán ser más adecuadas y más responsables (Rollano, 2004). Tanto el diseño, la innovación, así como la implementación de planes y programas en educación humanista, logran establecer con capacidad de diálogo y autoconocimiento, la cimentación de sujetos con calidad moral, para alcanzar la auténtica formación integral.

La educación centrada en procesos humanistas, permite restituir los procedimientos para el fortalecimiento de un determinado tipo de aprendizaje significativo y apropiarlos en la correspondencia a la educación humanista. Las acciones encaminadas a fortalecer la formación humanista, las cuales se formulen en la institución en diferentes trabajos, instituirán el tipo de formas que observarán la formación del estudiantado.

Es importante que las instituciones de educación superior sean sensibles al sentir de las personas estudiantes. Tradicionalmente, la educación ha estado más centrada en la presencia del profesorado, a pesar de que lo anterior es correcto, hace falta incluir la otra parte: la voz del estudiantado. En este sentido, como complemento a la reflexión teórica de este manuscrito, se aplicó un cuestionario a personas estudiantes universitarias, con la finalidad de conocer sus puntos de vista en torno a cómo aprecian ellos la intervención y la práctica del profesorado.

Al final de este documento, se incluyen algunos resultados y conclusiones en torno al paradigma del humanismo y en relación con cómo lo perciben los estudiantes. Desde luego, se incluyen las fuentes en las que se basaron cada una de las reflexiones conceptuales, motivo de este ensayo.

\section{DESARROLLO DEL TEMA}

En la historia de la educación, el proyecto que predomina es, en particular, el perfeccionamiento humano; mediante este se trasfieren conocimientos, acciones, hábitos y valores encaminados al bienestar social; por lo tanto, este constructo logra el establecimiento de las instituciones educativas. La educación, sin duda, es uno de los grandes retos de la sociedad.

Didriksson (2005) señala que la educación, y sustancialmente a la superior, le corresponde ser transformadora y a partir de un proceso creativo y compromiso pedagógico independiente debe impactar a la sociedad. Estas determinaciones precisan funciones de autoconstrucción de los estudiantes y el 
perfeccionamiento de prácticas para reconocerse como individuos activos, constructores y reformadores del conocimiento. De igual forma, al maestro le corresponderá desenvolver habilidades de un guía activo que auxilie la innovación para el perfeccionamiento humano, apropiada a las nuevas exigencias de la dinámica social.

Por otra parte, la Organización de las Naciones Unidas para la Educación, la Ciencia y la Cultura, UNESCO (1998), en la Declaración Mundial de la Educación Superior, subraya la importancia que este subsistema tiene en el avance sociocultural y económico. Las nuevas generaciones tienen que obtener los elementos teóricos y prácticos para constituirse en elementos transformadores de su entorno. Para ello, resulta necesario que adquieran conocimientos, habilidades y competencias, para ayudar al cambio educativo del presente y del futuro.

En esta misma línea, la Asociación Nacional de Universidades e Instituciones de Educación Superior, ANUIES (2000), en La educación superior en el siglo XXI, insiste en constituir líneas significativas de perfeccionamiento y de perspectiva sobre el sistema de educación superior, así como los rumbos por los cuales han de caminar las universidades, para de este modo plantearse los desafíos al presente con un enfoque prospectivo.

Desde luego que esta idea planteada en párrafos anteriores es compleja, debido a la situación por la que estamos viviendo en la actualidad, donde la incertidumbre y una sociedad líquida, como la prefiere llamar Bauman (2007). Líquida, en el sentido de que nada es estable, todo cambia sin que el ser humano esté realmente preparado para ello. Los acontecimientos se suceden demasiado rápido.

En cambio, para Rodríguez (2000), en un intento de dar una respuesta a este mundo un tanto caótico señala que lo incuestionable es que el cimiento de un sistema de educación superior es un trabajo nacional y los retos que tiene este nivel educativo solo conseguirán ser iniciados si se camina en dirección a una educación más cerca del sujeto, con todos los problemas que consigan proferir esta perspectiva.

\section{Algunos problemas actuales}

La educación en general y, de manera particular, la universitaria, está ante un mundo desconcertante: el resurgimiento de una forma más sutil, pero más eficiente de un sistema capitalista y neoliberal, trae bastantes retos a las universidades. Muñoz (2000) expone que concurren problemas que es importante enfrentar, como la deserción universitaria desde los primeros semestres, la reprobación y los bajos niveles de titulación. Estas, asociadas a los problemas de la falta de inclusión al campo laboral, conciben que la sociedad no debe minimizar las funciones de la educación superior. El papel de las universidades involucra no solo innovaciones de forma, sino de fondo significativas que admitan a la educación superior constituirse en una sólida columna para el país.

La globalización incide en las instituciones de educación superior por medio de la reducción de recursos públicos y procesos de privatización. Si bien las propuestas actuales no son ineficientes, pero sí lo son en contextos diferentes. México, con las desigualdades sociales, no puede permitirse un esquema de privatización de la educación.

En primer lugar, es necesario reducir las desigualdades (Brunner; 2001). Por otro lado, la perspectiva de los estudiantes universitarios ha cambiado; hace algunas décadas había cierta seguridad laboral para un egresado universitario. En cambio, actualmente todo es más azaroso, más de riesgo, debido a los cambios inesperados que se viven a nivel mundial.

Por tanto, las IES demandan evolucionar para la innovación, con ello se enaltece la calidad educativa en la producción y la transmisión de conocimientos. La universidad está obligada a convenir y 
desempeñar con las funciones procedentes del nuevo contexto mundial, para recobrar el reconocimiento de épocas pasadas.

Las estrategias de cambio son necesarias; el impulso de acciones para el beneficio de aprendizajes apropiados e innovadores para el discernimiento y la investigación son elementos clave. Para ello, el impulso de planes y programas institucionales, como la tutoría, son precisos en un proceso pedagógico selecto para conseguir aprendizajes adecuados para la vida.

\section{Una posibilidad: los programas universitarios de tutorías}

Ante la necesidad de perfeccionamiento de los procesos de la educación superior, la Asociación Nacional de Universidades e Instituciones de Educación Superior (ANUIES) (1999), y al reconocer la urgencia de adopción de estrategias concretas dirigidas a la atención individual e integral de los estudiantes, plantea considerar el Programa Institucional de Tutoría (PIT) como el conjunto de acciones encaminadas para apoyar a los estudiantes durante su trayecto escolar en las instituciones educativas, con el fin de proporcionarles dispositivos académicos en los aspectos que influyen en su desempeño académico, logro profesional, que la conceptualiza como un proceso de acompañamiento durante la formación de los estudiantes, se sintetiza mediante el cuidado individualizado a un estudiante o a un grupo reducido de estudiantes, por parte de académicos voluntarios, formados para esta función, apoyándose conceptualmente en las teorías del aprendizaje, más que las de enseñanza.

La oferta de la ANUIES con el PIT, intenta abatir los índices de reprobación y rezago escolar, reducir las tasas de deserción y elevar la eficiencia terminal. A partir de 2000, gran parte de las instituciones de la educación superior desplegaron proyectos y acciones de tutoría, desde luego, con resultados diferentes, debido a que las acciones de la tutoría son versátiles, según los contextos, los rasgos corporativos y los colaboradores; por lo cual Baudrit (2000) la observa como un formulario de usos y variedades múltiples. En el ámbito educativo, los tutores han transitado a partir de la instrucción en áreas con problemas para el aprendizaje, hasta como consejeros y orientadores en el proceso educacional.

El principio de la tutoría se inicia en los años sesenta del siglo XX en los países europeos. Para Topping (1989), los actores del proceso siempre han sido el tutor, el tutorado y el proceso tutorial en sí mismo; no obstante, tendría que registrar la atribución que asumen en el proceso elementos como el currículum, la institución y el modelo académico, entre otros. En México, desde el siglo XIX surge la figura y el monitor y el tutor en el contorno educativo con los estudiantes de mayor rendimiento académico, con el propósito de educar a sus similares en las áreas del conocimiento, apoyando a los compañeros de niveles inferiores, así como a los de mayor insuficiencia académica.

La experiencia descrita surge precisamente con el modelo pedagógico denominado Escuela lancasteriana; solo era necesario un profesor para un grupo de hasta cien alumnos; pues los monitores se encargaban de ir compartiendo lo que aprendían con el resto. La escuela lancasteriana no llegó a triunfar más allá de la enseñanza primaria.

En gran parte de las universidades y las IES, la estrategia de tutoría logra un avance significativo y privilegia el tipo llamado tutor, consultor o asesor, cuyas particularidades se consideran en la siguiente concepción propuesta por Rodríguez (2004), quien señala que la tutoría es un método de atención a estudiantes que se ocupan de la investigación, formación y orientación personalizada y centra su cuidado en proporcionar la adaptación a la universidad, el proceso de aprendizaje, perfeccionar el rendimiento académico; asimismo como situar la elección curricular y profesional.

Desde esta perspectiva, García Nieto (2005) señala que la tutoría es un proceso formativo elaborado por el profesor-tutor, enfocado al perfeccionamiento integral, profesional, intelectual y humano de los 
estudiantes. La figura de profesor-tutor significa que una persona profesora universitaria, de medio o tiempo completo, realiza, además de sus actividades docentes, un trabajo extraordinario; auxiliar a los estudiantes en su vida académica mediante programas específicos de tutorías.

La función de guiar al alumnado durante el tránsito universitario, se efectúa por medio de acciones heterogéneas: entrevistas, llenado de formatos de datos particulares para concertar expedientes de las contextos socioeconómicos, familiares y afectivos, como coadyuvantes en la localización de problemáticas extra académicas, así como concertar responsabilidades y horarios para las sesiones de trabajo. Las acciones se efectúan con la intención de ubicar al estudiante hacia programas de apoyo institucional: asesoría académica, atención psicológica, psicopedagógica, alimentación y médica, principalmente.

En ese sentido, se piensa que la estrategia tutorial requiere interesarse también en los contenidos de los cursos académicos, con el fin de proporcionar un camino al tipo de tutoría complementaria, que conseguirá ser efectuada en combinación entre las figuras de tutores docentes y tutores entre iguales de mayor rendimiento académico que, en esta forma clásica, se benefician los métodos de comunicación, la confianza recíproca y la estimulación a los estudiantes (tutelados) superar las dificultades escolares. Habrá que insistir y trabajar más en las academias de profesores para comprender y hacer suyos aspectos sustanciales de las tutorías universitarias.

Un aspecto importante es la relación de hermandad entre personal docente-tutor y tutores para conseguir el rendimiento para el éxito del programa (Baudrit, 2000); pues la parte subjetiva ha de ser manejada de la mejor manera. Quizá la relación afectiva entre los estudiantes y el profesor y tutor es la clave del éxito. En la tutoría entre pares es interesante contar con el soporte de un compañero, al mismo tiempo trabajar conocimientos y habilidades, la información de percepciones y métodos en un clima de pares, ayuda a formar compañerismo en el tutelado y, al tutor-estudiante, le permite desarrollar sus métodos cognitivos que favorece al bien de otros (Vadillo, 2011). En esta dirección se justifica, puesto que la tutoría se ha pensado como el asunto de acompañamiento en la formación integral del estudiante, con la intención primordial de reconocer al nuevo paradigma centrado en la forma cómo consiguen los conocimientos, las habilidades y las actitudes de los estudiantes.

En este aspecto, Castellanos, Venegas y Ramírez (2003) expone que, en los sistemas institucionales, la tutoría desenvuelve un enfoque integral y humanista; por lo tanto, considera necesario la formación de valores en el aprendizaje del estudiante. Las instituciones universitarias se colocan en otras vías para desarrollar la estrategia tutorial y la curricular, que por medio de trayectorias buscan formar y defender el paradigma del aprendizaje, el cual esté indeleble y para el progreso del ser humano.

De acuerdo con la definición de Vadillo (2011), el docente debe concebir el curso en el tejido global de toda acción de aprendizaje en que quedará sumergido el estudiante; es señalar, que cada curso le corresponderá relacionarse con los demás, de tal manera que la transdisciplinariedad posee como objetivo crear el perfil de egreso ambicionado, al mismo tiempo subrayar tanto el desarrollo de contenidos (conocimientos expresivos) como de prácticas de forma y de actitudes.

Además, la vía institucional, específica por sus adecuadas experiencias formativas, influye en la cimentación de sus estudiantes, de forma que el ambiente áulico, la cual es la variable que da más cuenta del provecho escolar, es el clima que se vive en el salón; mientras las reglas y su cultura quebrantan en la formación de hábitos y actitudes que serán precisas en la formación exhaustiva de los sujetos.

En esta perspectiva se toma en cuenta los aspectos tanto a nivel personal como las relaciones interpersonales, en unión con las inicialmente detalladas. Todos estos relativos admiten marcar los elementos clave para la maniobra metodológica del asunto tutorial, en el objetivo de la formación integral de los estudiantes. El curriculum, el diseño, la actualización e implementación de planes y programas facilitarán y mejorarán los aprendizajes significativos de los estudiantes. 
En el marco humanista se promueve el desarrollo integral de la persona: aspectos físicos, mentales, espirituales y sociales con sustento en valores que son aceptados por todos. La educación centrada en los estudiantes plantea estrategias para la solución de dificultades hacia la deferencia de los contenidos requeridos para el beneficio de aprendizajes pertinentes y significativos. Conseguir que el estudiante valore y formalice las normas sociales, como elementos necesarios para la formación de los individuos independientes, es una de las metas de una concepción humanista de la educación. La formación de estudiantes comprometidos consigo mismos y los demás es una tarea de la formación en valores. Es justamente en estos aspectos que le paradigma pedagógico humanista hace hincapié de manera reiterada.

\section{El enfoque de competencias para las tutorías universitarias}

Transformar esencialmente la ecuación conocimiento-docente-estudiante, se pasa de los saberes a las competencias será viable si se toma conciencia, se rediscute y se retornan los ejes elementales que subyacen al presente modelo de educación (Aguerrondo,1993). El enfoque por competencias tiene como fin el perfeccionamiento de estudiantes autónomos que se autoconstruyan, los cuales asimilen a aprender haciéndolo de manera eficaz y apropiada. Las innovaciones en los procesos educativos de la educación superior requieren nuevas disposiciones que soportan el cambio de paradigma de la experiencia educativa.

La transformación en la forma de enseñar y aprender son hechos incuestionables influidos por la mejora tecnológica, que propicia una nueva forma de acción del proceso didáctico, el establecimiento de las relaciones interpersonales y el de los valores, los cuales son recapitulaciones fundamentales del nuevo modelo educativo para disminuir el fracaso escolar. El enfoque por competencias en el ámbito universitario no está en contradicción con el humanismo. El desarrollo humano comprende diversas dimensiones que se constituyen e integran en la interacción con el medio cultural. Las competencias humanas son una forma de conciencia, producto de la integración de conceptos, destrezas y actitudes, que dota al ser humano de una capacidad de entendimiento, acción y transformación de sus relaciones con el mundo.

El enfoque de formación de competencias, de acuerdo con Pimienta (2012), como el trabajo integral de la persona estudiante, que involucra conocimientos, habilidades, destrezas, actitudes y valores dentro de un contexto ético, sostiene que en este enfoque alcanzará las operaciones para solucionar dificultades para la vida. La educación centrada en el aprendizaje subraya la innovación en la forma para adquirir los contenidos, en el cual se privilegia el contexto. Ello admitirá mover nuevos conocimientos, habilidades, destrezas y actitudes para interponerse en la solución de escenarios problemáticos, con el resultado de mejora o generación de nuevas competencias.

La acción tutorial, como parte de la tarea educativa, ha destacado la acción de la instrucción, como anota Martín, Puig, Padrós, Rubio y Trilla (2003), al reseñar especialmente al aprendizaje de conocimientos y adquisición de habilidades y destrezas rentables para la vida. No obstante, también cree que debe administrar su trabajo a los procesos pedagógicos, ya que esta subraya los aspectos de formación en valores que inciden en la personalidad de los tutorados, sus relaciones con los restantes y la sociedad.

\section{El humanismo en el aula universitaria}

Desde el punto de vista humanista, la educación está interesada en ayudar a los estudiantes para que decidan lo que ellos son y lo que quieren llegar a ser. La educación humanista se basa en la idea de que todos los sujetos son diferentes y los ayuda a ser más como ellos mismos y menos como los demás. Para Hamachek, citado por Hernández (1997) es necesario auxiliar a los estudiantes a explorar y comprender 
más adecuadamente el conocimiento de su persona y los significados de sus experiencias vivenciales. Esta idea ha de buscar formatos dentro del aula y fuera de la misma.

La educación debe tener un sentido para cada estudiante: hacia dónde van, qué desean de la vida, para qué estudian determinada carrera universitaria. Pero este sentido ha de construirse con bases firmes y considerar la realidad que vive cada uno de ellos. En este sentido, es importante la presencia en el aula del profesor, como el sujeto que inspira que muestra al otro no aquello que debe hacer, sino el camino que él mismo ha recorrido. Para Rogers (1978) la educación debiera fomentar el aprendizaje significativo y vivencial que involucra a la persona total del alumno.

La educación debe recrear un clima de libertad total para que el alumno se sienta en un ambiente educativo, lo cual favorece la realización de él no solo como estudiante o parte de una universidad, sino sustancialmente como sujeto. Esta parte es la esencia de este paradigma humanista; lograr que el estudiante vea más allá del aula y la institución, que se vea en un futuro inmediato como una persona diferente.

En la educación humanista el docente permite que los alumnos aprendan, mientras impulsa y promueve todas las exploraciones, experiencias y proyectos que estos preferentemente inicien o decidan emprender con el fin de conseguir aprendizajes vivenciales con sentido. De acuerdo con el paradigma humanista, los alumnos son entes individuales, únicos, diferentes de los demás; personas con iniciativa, con necesidades personales de crecer, con potencialidad para desarrollar actividades y solucionar problemas creativamente. Las nuevas reformas universitarias insisten en esa parte subjetiva del proceso educativo. El docente ha de estar reconciliado con el adolescente o el joven que fue, para que de esta manera esté en mejores condiciones de ayudar a sus estudiantes (Rodríguez, 2000).

Los estudiantes no son seres que solo participan cognitivamente, sino personas con afectos, intereses y valores particulares, a quienes debe considerarse en su personalidad total. Gobernar almas no es el propósito final del docente humanista, sino formar a los estudiantes en la toma de decisiones dentro de ámbitos, en los cuales prime el respeto a los derechos de la persona y lo justo y lo injusto, como dogma, se cuestione. Cambiar a nuevos esquemas pedagógicos siempre es complicado, los docentes, tienen una larga tradición en sus formas de llevar a cabo su acción educativa. Luego entonces, es necesario señalar algunos de los rasgos que debe asumir el educador dentro del contexto humanista, de acuerdo con lo presentado por González (2004):

a) Ha de ser un maestro interesado en el alumno como persona total.

b) Procura mantener una actitud receptiva hacia nuevas formas de enseñanza.

c) Fomenta en su entorno el espíritu cooperativo.

d) Es auténtico y genuino como persona, y así se muestra ante sus alumnos.

e) Intenta comprender a sus estudiantes poniéndose en el lugar de ellos (empatía).

f) Actúa con mucha sensibilidad hacia sus percepciones y sentimientos.

f) Rechaza las posturas autoritarias y egocéntricas.

g) Pone a disposición de los alumnos sus conocimientos y experiencia.

Estas proposiciones son básicas en el devenir de los maestros universitarios en el aula. El humanismo implica estas consideraciones pedagógicas que debe tener en cuenta el docente, cuando está en relación pedagógica con cada uno de sus estudiantes. Desde luego, para muchos profesores no es fácil hacer este tipo de cambio, pero con trabajo colegiado se puede conseguir resultados que mejoren la calidad del trabajo áulico. 
El paradigma humanista en la educación, señala Hernández (1998), hace una crítica a la educación tradicional como partidaria de la enseñanza directa y rígida, predeterminada por un currículo inflexible y centrada en el profesor. El humanismo vuelve a mirar al estudiante como un sujeto que siente, vive un contexto determinado y está marcado por una historia que es individual, el que vive intensamente su experiencia dentro del contexto universitario.

\section{Percepciones de los estudiantes sobre sus maestros}

Basado en lo anterior, es importante conocer la percepción del alumnado acerca del profesoresado en este ámbito. Para ello, se llevó a cabo un estudio de tipo diagnóstico basado en cuestionario aplicado a 60 personas estudiantes del quinto semestre, para conocer su percepción y saber en qué asignaturas consideran que la persona docente es predominantemente humanista. Al analizar la información se muestra que perciben que aún falta que el profesor se sustente en el humanismo. Un $86 \%$ de los jóvenes indica que todos los profesores en algunas sesiones sí muestran características humanistas, pero el resto es tradicional. Desde luego que en un primer momento se les explicó qué era el humanismo y las características de un profesor bajo este enfoque, y así se procedió a que ellos dieran a conocer su percepción.

En función a las asignaturas que consideran que ya emplean esta modalidad en su mayoría de sesiones, mencionan que son: "Metodología de la Investigación", "Ecología", "Ciencias de la Salud" y "Psicología". El factor que indican no observar en ningún profesor es que no perciben que los docentes disfruten dar sus clases, factor relevante para considerar con los compañeros docentes para mejorar en medida de lo posible. Este elemento llamó la atención. Los estudiantes son muy perceptibles a los estados de ánimo de los maestros. Esta idea es válida desde los niveles de preescolar, hasta los ámbitos universitarios. El maestro trabaja con su cuerpo, sus afectos y sus emociones.

Una vez que se analiza la información cualitativa que ofrecen los jóvenes, se perciben que aún falta que el profesor se sumerja en el humanismo; un $86 \%$ de los jóvenes indica que todos los profesores en algunas sesiones sí muestran características humanistas, pero el resto de ellos vuelve a ser tradicional. En función a las asignaturas que consideran que ya emplean esta modalidad en su mayoría de sesiones, mencionan que son "Metodología de la Investigación", "Ecología", "Ciencias de la Salud" y "Psicología". Los estudiantes señalan que no observaron a ningún profesor disfrutar sus clases, factor relevante para considerar con los compañeros docentes para mejorar en medida de lo posible. Este aspecto llama la atención; el ambiente áulico debe ser óptimo, con cierta calidez y hasta un tanto divertido, lo cual no significa, desde luego que se pierda la seriedad con la que deben tratarse todos los temas en el aula.

\section{SÍNTESIS Y REFEXIONES FINALES}

A lo largo de este documento se ha planteado la necesidad de hacer ciertas recuperaciones del orden pedagógico. Las universidades con sus esquemas muchas veces descontextualizados, en los cuales reina la rigidez de los programas y la presencia, casi absoluta del profesor, se hace necesario hacer una "revolución" en el sentido de establecer las bases para una educación centrada más en el estudiante. En los paradigmas pedagógicos surge el denominado paradigma humanista, quien pone el acento en el estudiante como persona. Desde luego, que lo anterior no significa hacer a un lado la figura del profesor, pero sí poner más énfasis en el estudiante. El esquema, muchas veces rígido de la universidad ha de dar paso a una flexibilización pedagógica.

Para ello, instituciones como ANUIES han propuesto diversas opciones de cambio entre los que se pueden destacar los programas de tutorías. Es preciso, para la educación superior, abordar con eficiencia 
los procesos de acompañamiento de los estudiantes con la tutoría como estrategia para regular sus procesos de aprendizaje, estancia, permanencia y culminación exitosa. También, la estrategia de educar en valores es apreciada como tema notable para la construcción integral de los sujetos que, en la actualidad, se enfrentan a retos cada vez más complejos en este siglo XXI. (Brovetto; 2000).

Los aspectos de educación y formación no consiguen reflexionarse independientes, más bien son sumados del proceso educativo en el beneficio de una formación integral. No obstante, se considera necesario atender especialmente los aspectos formativos porque la formación o la educación en valores es necesaria en la construcción de la personalidad moral y la formación de una ciudadanía responsable y, además, porque la formación es indispensable para lograr la instrucción educativa.

En otro término, no hay instrucción sin la educación en valores. En el nuevo modelo educativo universitario los valores y la tutoría; además de otros procesos educacionales, constituyen elementos clave para lograr una educación plena que responda a los estándares de las expectativas de la sociedad contemporánea y el futuro. Mirar al estudiante como un sujeto con deseos, esperanzas, pero también con incertidumbres es el reto que los profesores universitarios deben asumir.

\section{REFERENCIAS}

Aguerrondo, I. (1993). La calidad de la educación, ejes para su definición y evaluación. Revista Interamericana de Desarrollo Educativo, (116).

ANUIES (1999). La educación superior en el siglo XXI. Líneas estratégicas de desarrollo. Una propuesta de la anuies. México: ANUIES.

ANUEIS (2000). Programas institucionales de tutorías. Una propuesta de la anuies para su organización y funcionamiento en las instituciones de educación superior. México.

Baudrit, S. (2000). Nuevos esquemas educativos; el humanismo en la educación. Madrid: Editorial Narcea.

Bauman, Z. (2007). Tiempos líquidos. Vivir en una época de incertidumbre. España: Tusquest editores.

Brovetto, J. (2000). La educación superior para el siglo XXI. En Tünnermann, C. y López, F. La educación en el horizonte del siglo XXI. Caracas: IESALC/UNESCO.

Brunner, J. (2001). Globalización y el futuro de la educación. Tendencias, desafíos, estrategias en análisis de prospectivas de educación en américa latina y el caribe Santiago: UNESCO.

Castellanos, A., Venegas, F. y Ramírez, J. (2003). Sistemas tutoriales en el centro occidente de México. México.

Didriksson, A. (2005). La universidad de la innovacion. Una estrategia de transformacion para la construccion de universidades del futuro. España: Plaza y Valdés.

González, E. (2004). La psicología clínica de la "tercera fuerza", en el contexto internacional: estados unidos y alemania". Editorial Mimeo.

García Nieto, N. (2005). "El papel de la tutoría en el espacio europeo de educación superior". En Chamorro, M. y Sánchez, P. Iniciación a la docencia universitaria. Manual de ayuda. Madrid: Instituto de Ciencias de la Educación de la Universidad Complutense. 23

Hernández, G. (1997). Módulo fundamentos del desarrollo de la tecnología educativa (bases psicopedagógicas). México: ILCE-OEA.

Hernández, G. (1988). Paradigmas en psicología de la educación. México. Ed. Paidós.

Neill, A. (1979). Corazones no solo cabezas en la escuela. México: Editorial Fondo de Cultura Económica. 
Muñoz, A. (2000). Técnicas psicoeducativas para la intervención en la mejora de la inteligencia moral, Simposio de programas de intervención cognitiva. Granada, 5-8 de abril.

Papalia, D.; Martorell, G. y Feldman, R. (2006). A child's world: infancy through adolescence. New York: McGraw-Hill.

Pimienta, J. (2012). Las competencias en la docencia universitaria. México: Editorial Pearson Educación.

Martín, X., Puig, J., Padrós, M., Rubio, L. y Trilla, J. (2003). Ser tutor/a. Barcelona: Fundación Jume Bofill. Recuperado de https://fundaciobofill.cat/uploads/docs/3/t/8/b/t/3/0/s/s/1342.pdf

Rogers, C. (1978). Orientación psicológica y psicoterapia. Madrid: Ed. Narcea.

Rodríguez, R. (2000). La reforma de la educación superior. Señas del debate internacional a un siglo. Revista Electrónica de Investigación Educativa. 2 (1), México: Universidad Nacional Autónoma de México.

Rodríguez, S. (coord.) (2004). Manual de tutoría universitaria. Recursos para la acción. Barcelona: Editorial Octaedro.

Rollano, D. (2004). Educación en valores: cómo enfocar la educación hacia la ética. Madrid; Editorial Ideas Propias.

UNESCO (1998). Declaración Mundial de la Educación Superior. Recuperado de https://www.iesalc. unesco.org/ess/index.php/ess3/article/view/171

Topping, K (1989). Peer tutoring and paired reading: combining two powerful techniques. The Reading Teacher. 42 (7):488-494. Recuperado de https://www.jstor.org/stable/20200196?seq=1

Vadillo, G. (2011). De maestro a tutor académico. Cuarenta semanas de clases innovadoras y efectiva. México: Editorial Paidós. 